\title{
Combined Enteral and Parenteral Glutamine Supplementation in Endotoxemic Swine: Effects on Portal and Systemic Circulation Levels
}

\author{
George Stavrou ${ }^{a, b} \quad K^{b}$ stantinos Arvanitidis ${ }^{c}$ Eirini Filidou $^{c}$ \\ Kyriakos Fotiadis $^{a}$ Vasilios Grosomanidis ${ }^{a}$ Aris loannidis ${ }^{a}$ Georgia Tsaousi $^{a}$ \\ Antonios Michalopoulos $^{a} \quad$ George Kolios $^{c}$ Katerina Kotzampassi ${ }^{a}$ \\ ${ }^{a}$ Department of Surgery, Aristotle University of Thessaloniki, Thessaloniki, Greece; ${ }^{\text {b}}$ York Teaching Hospital NHS \\ Foundation Trust, York, UK; ' ${ }^{2}$ Laboratory of Pharmacology, Faculty of Medicine, Democritus University of Thrace, \\ Alexandroupolis, Greece
}

\section{Significance of the Study}

- The findings of the present study in an endotoxemic swine model indicate that combined enteral and parenteral glutamine (GLN) supplementation confers significant superiority over intravenous supplementation alone, in terms of enhanced availability in systemic and portal circulation during the course of endotoxemia. Thus, combined GLN treatment is a beneficial practice, ensuring adequate GLN to compensate for the resulting intracellular shortage.

\section{Keywords}

Glutamine · Endotoxemia

\begin{abstract}
Objective: To measure plasma glutamine (GLN) levels in systemic and portal circulation after combined enteral and parenteral administration in early endotoxemic swine. We hypothesized that this combination will be more efficient than intravenous administration alone in restoring plasma levels during the course of endotoxemia. Materials and Methods: Endotoxemia was induced with Escherichia coli O111:B4 lipopolysaccharide (LPS) $(250 \mu \mathrm{g} / \mathrm{kg}$ body weight) in 16 anesthetized, fasted swine and maintained by constant infusion ( $2 \mu \mathrm{g} / \mathrm{kg} / \mathrm{h}$ ) over $180 \mathrm{~min}$. Another 16 swine served as controls. After infusion with LPS or placebo, GLN was administered intravenously, enterally or in combination $(0.5 \mathrm{~g} / \mathrm{kg}$ i.v.
\end{abstract}

plus $0.5 \mathrm{~g} / \mathrm{kg}$ enterally) over $30 \mathrm{~min}$. At 0, 15, 30, 45, 60, 120 and $180 \mathrm{~min}$, blood was drawn from the systemic and portal circulation for colorimetric assessment of GLN. Results: In healthy, placebo-alone swine, GLN levels remained stable throughout the study. Intravenous and combined infusion increased systemic levels $(p=0.001)$, but after enteral administration alone, a smaller effect was observed $(p=0.026)$. Portal levels were increased after combined, enteral and intravenous administration ( $p=0.001)$. In endotoxemia, systemic and portal levels decreased significantly. Intravenous and, to a greater extent, combined administration increased systemic levels ( $p=0.001$ ), while enteral administration only had a small effect $(p=0.001)$. In the portal vein, intravenous and combined treatment increased plasma levels $(p=0.001)$, whereas enteral supplementation alone had again a small, yet significant effect $(p=0.001)$. Conclusions: The findings indicate that combined GLN supplementation is superior to

\begin{tabular}{ll}
\hline KARGER & $\begin{array}{l}\text { ( ) 2018 The Author(s) } \\
\text { Published by S. Karger AG, Basel }\end{array}$ \\
E-Mail karger@karger.com & $\begin{array}{l}\text { This is an Open Access article licensed under the Creative Commons } \\
\text { Attribution-NonCommercial-4.0 International License (CC BY-NC) } \\
\text { (http://www.karger.com/Services/OpenAccessLicense), applicable to } \\
\text { the online version of the article only. Usage and distribution for } \\
\text { commercial purposes requires written permission. }\end{array}$
\end{tabular}

George Stavrou

York Teaching Hospital

NHS Foundation Trust

York YO318HE (UK)

E-Mail stavgd@gmail.com 
intravenous treatment alone, in terms of enhanced availability in systemic and portal circulations. Thus, combined treatment at the onset of endotoxemia is a beneficial practice, ensuring adequate $\mathrm{GLN}$ to compensate for the resulting intracellular shortage.

(c) 2018 The Author(s)

Published by S. Karger AG, Basel

\section{Introduction}

In catabolic states an increased release of glutamine (GLN) from the skeletal muscle to meet the increasing demands of peripheral tissue is observed as part of the body's conserved evolutionary response to stress, progressively leading to a depletion of GLN, proportional to the severity and duration of illness [1-3].

Various studies in endotoxemia/sepsis models $[3,4]$ have reported the depletion of GLN and its consequences on body homeostasis and progress of disease. However, it has not been fully elucidated whether parenteral or enteral administration may be more beneficial in restoring GLN stores - in the same model - as, to the best of our knowledge, there are no studies regarding the effect of exogenous GLN administration on plasma levels and, especially, relating to the route of administration.

The present experimental study was designed to measure plasma GLN levels in systemic and portal circulation after enteral, parenteral or combined administration in a healthy and early-stage endotoxemia swine model. We hypothesized that the combination would be more efficient in restoring plasma levels during the course of endotoxemia.

\section{Materials and Methods}

The experiments were performed at the Surgical Research Laboratory of the AHEPA Hospital (Aristotle University of Thessaloniki) and the Laboratory of Pharmacology (Democritus University of Thrace), Greece. The experimental protocol was approved by the Department of Animal Care and Use Committee of the Greek Ministry of Agriculture, according to the European Community Guiding Principles for the Care and Use of Animals (EU Directive 2010/63/EU, Protocol No. 164909/11.05.2012).

\section{Setting}

Following $18 \mathrm{~h}$ of food deprivation, 33 female Munich swine (3 months old, $23.0 \pm 2.9 \mathrm{~kg}$ body weight) were premedicated, intubated and mechanically ventilated, as previously described $[5,6]$. The femoral artery and both femoral veins were cannulated; a 7.5Fr Swan-Ganz CCOmboV catheter, a 20-Fr gastrostomy tube (for GLN administration) and a urinary catheter were placed invasively as well as a portal vein catheter through the upper mesenteric vein.
Experimental Model

Escherichia coli endotoxin lipopolysaccharide (LPS) (0111:B4, L2630, Sigma-Aldrich Co. LLC, St. Louis, USA) was used in a bolus dose of $250 \mu \mathrm{g} / \mathrm{kg}$ body weight in $20 \mathrm{~mL} 5 \%$ dextrose followed by a constant infusion of $2 \mu \mathrm{g} / \mathrm{kg} / \mathrm{h}$, throughout the experiment, to simulate the effects of endotoxemia in terms of its hemodynamic and metabolic consequences $[5,6]$.

\section{Experimental Design}

The animals were randomized to receive, through the Swan-Ganz catheter, either LPS (endotoxemia group) or dextrose (control group) at time 0 (baseline). Thereafter, both groups were subdivided into: control, intravenous, enteral, and combined intravenous + enteral subgroups; all groups were given GLN intravenously and/or enterally, or placebo treatment (intravenously and/or enterally) for the next $30 \mathrm{~min}$, which were provided through the ipsilateral femoral vein as the LPS and/or gastrostomy, respectively (Table 1).

\section{GLN Assay}

Blood samples were collected from the arterial and portal circulation in EDTA tubes at baseline and at 15, 30, 45, 60, 120 and $180 \mathrm{~min}$, for a colorimetric assay of GLN, using the EnzyChrom ${ }^{\mathrm{TM}}$ Kit (BioAssay Systems, Hayward, CA 94545, USA). Briefly, two distinct reagent mixes were prepared: a "working mix", containing both enzymes supplied, and a "blank mix", with only the second enzyme. The values obtained from the working mix represented total GLN and glutamate concentrations, while those from the blank mix showed the concentration of glutamate in the sample. The results for GLN concentration were calculated via a standard curve, after subtracting the optical density values from the "blank mix", and corrected for the presence of intracellular glutamate, as recommended by the manufacturer.

\section{Statistical Analysis}

An estimated difference in plasma GLN levels of $\geq 0.1 \mathrm{mmol} / \mathrm{L}$ (standard deviation $0.02 \mathrm{mmol} / \mathrm{L}$ ) between enteral and enteral + parenteral GLN supplementation groups in the endotoxemia setting was defined as the primary end point. Therefore, a sample size of 4 animals in each group was estimated for a type 1 error of 0.05 $(\alpha=0.05)$ and a power of $80 \%$. Normality of data was assessed by the Shapiro-Wilks test. Analysis of variance for multifactor repeated measures with Greenhouse-Geisser correction was conducted to analyze continuous variables over time. If variance/covariance matrices were not equal across all groups and if overall sphericity for each group was violated, then we used contrasts for between/ within tests and Tukey HSD adjustment. Values are presented as means and standard deviations. A $p$ value $<0.05$ was considered significant. The Statistical Package for Social Sciences (SPSS, version 19.0; SPSS Inc., Chicago, IL, USA) was used.

\section{Results}

In healthy swine there was no difference in plasma GLN levels, both in systemic and portal circulations, throughout the 180-min study period, while in LPS-treated ones, a progressive, statistically significant decline ( $p=$ 0.001), compared to baseline, was observed (Fig. 1). 
Fig. 1. Time course of glutamine (GLN) levels in systemic and portal circulation in control and endotoxemic swine (not GLNtreated). Data are expressed as means \pm standard deviation. ${ }^{*} p<0.05,{ }^{* *} p<0.01$, $* * * \quad p<0.001$ represent significance of endotoxemia (mentioned as "sepsis") systemic and portal groups compared to control systemic and portal groups, respectively.

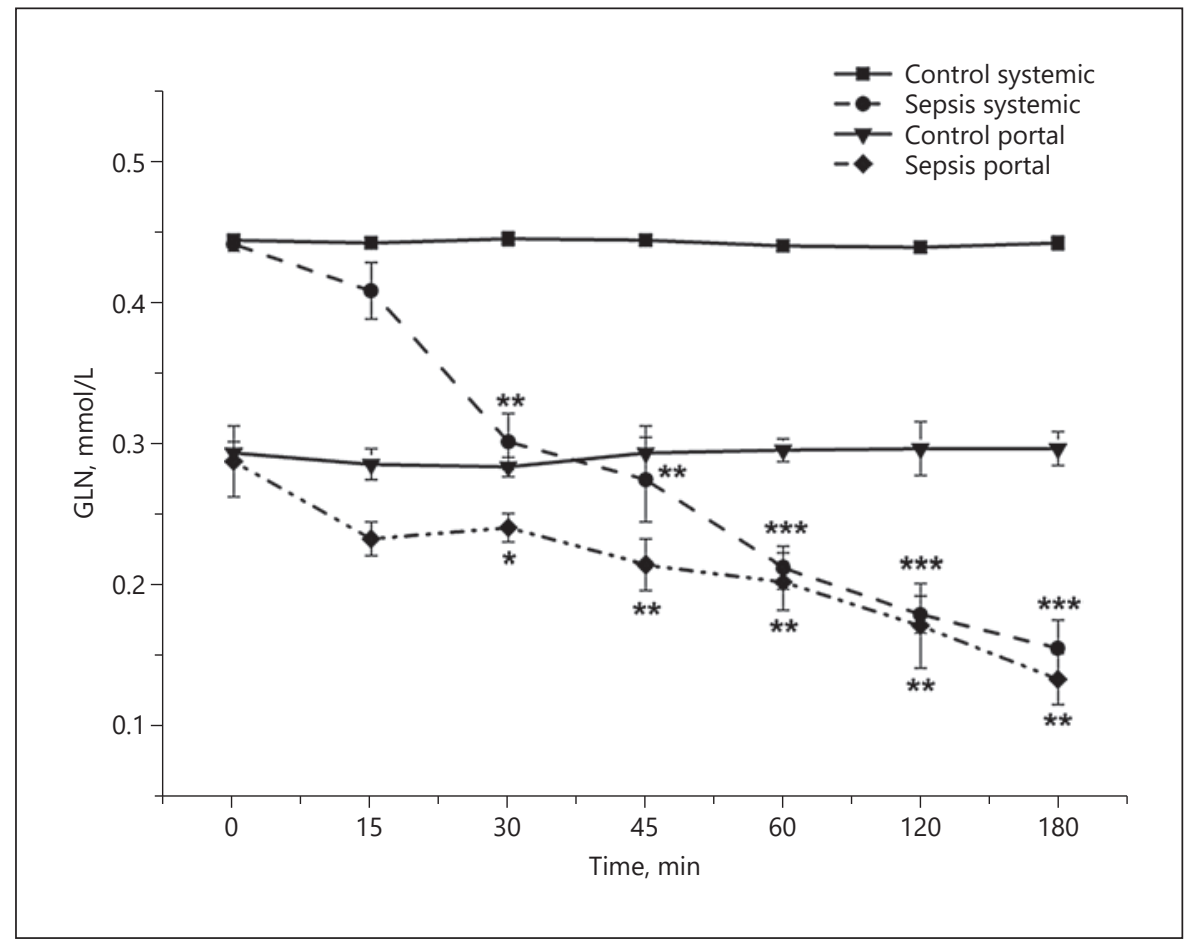

Table 1. Study groups

\begin{tabular}{|c|c|c|}
\hline Groups & $\begin{array}{l}\text { Healthy animals } \\
(20 \mathrm{~mL} 5 \% \text { dextrose })\end{array}$ & $\begin{array}{l}\text { Endotoxemic animals } \\
(250 \mu \mathrm{g} / \mathrm{kg} \mathrm{BW}+2 \mu \mathrm{g} / \mathrm{kg} / \mathrm{h} \text { LPS })\end{array}$ \\
\hline \multicolumn{3}{|l|}{ Control } \\
\hline Intravenous & $100 \mathrm{~mL}$ normal saline $0.9 \%$ & $100 \mathrm{~mL}$ normal saline $0.9 \%$ \\
\hline Enteral & $100 \mathrm{~mL}$ tap water & $100 \mathrm{~mL}$ tap water \\
\hline \multicolumn{3}{|l|}{ Intravenous } \\
\hline Intravenous & Ala-Gln dipeptide $0.5 \mathrm{~g} / \mathrm{kg} \mathrm{BW}^{1}$ & Ala-Gln dipeptide $0.5 \mathrm{~g} / \mathrm{kg} \mathrm{BW}^{1}$ \\
\hline Enteral & $100 \mathrm{~mL}$ tap water & $100 \mathrm{~mL}$ tap water \\
\hline \multicolumn{3}{|l|}{ Enteral } \\
\hline Intravenous & $100 \mathrm{~mL}$ normal saline $0.9 \%$ & $100 \mathrm{~mL}$ normal saline $0.9 \%$ \\
\hline Enteral & $0.5 \mathrm{~g} / \mathrm{kg}$ BW GLN powder ${ }^{2}$ & $0.5 \mathrm{~g} / \mathrm{kg}$ BW GLN powder ${ }^{2}$ \\
\hline \multicolumn{3}{|c|}{ Intravenous + enteral } \\
\hline Intravenous & Ala-Gln dipeptide $0.5 \mathrm{~g} / \mathrm{kg} \mathrm{BW}^{1}$ & Ala-Gln dipeptide $0.5 \mathrm{~g} / \mathrm{kg} \mathrm{BW}^{1}$ \\
\hline Enteral & $0.5 \mathrm{~g} / \mathrm{kg}$ BW GLN powder ${ }^{2}$ & $0.5 \mathrm{~g} / \mathrm{kg} \mathrm{BW}$ GLN powder $^{2}$ \\
\hline \multicolumn{3}{|c|}{$\begin{array}{l}\text { BW, body weight. }{ }^{1} \text { Alanine-GLN (Ala-Gln) dipeptide, Dipeptiven }{ }^{\circledR} \text {, Fresenius Kabi Hellas SA, Athens; } \\
13.5 \mathrm{~g} \text { GLN/100 } \mathrm{mL} ; 0.5 \mathrm{~g} / \mathrm{kg} \mathrm{BW} \text { of Ala-Gln dipeptide, equivalent to } 0.35 \mathrm{~g} / \mathrm{kg} \text { BW GLN, as per clinical guide- } \\
\text { lines, infused over } 30 \mathrm{~min} .{ }^{2} \text { GLN Plus }{ }^{\circledR} \text {, Fresenius Kabi Hellas SA, Athens, } 44.6 \mathrm{~g} \text { GLN/100 g, diluted in equal } \\
\text { volume of tap water, administered over } 30 \text { min. }\end{array}$} \\
\hline
\end{tabular}

Endotoxemia was identified by a rise in the pulmonary artery pressure $(33.3 \pm 8.3 \mathrm{~mm} \mathrm{Hg}$ in endotoxemic vs. $18.2 \pm 0.5 \mathrm{~mm} \mathrm{Hg}$ in healthy animals, $p<0.001)$ as well as by leukopenia $(4,733 \pm 3,442 \mathrm{WBC} / \mathrm{L}$ in endotoxemic vs. $12,048 \pm 1,506 \mathrm{WBC} / \mathrm{L}$ in healthy animals, $p=0.003)$.
No hemodynamic changes were noted after GLN administration.

In healthy swine, after intravenous GLN administration, systemic plasma levels peaked (36-fold) at $30 \mathrm{~min}$ $(p<0.001)$, followed by a progressive reduction (15-fold 
Fig. 2. a Time course of glutamine (GLN) levels in systemic circulation in GLN-treated control swine. Data are expressed as means \pm standard deviation. ${ }^{* * *} p<0.001$ represents significance of GLN intravenous and GLN intravenous + enteral groups compared to both control systemic and GLN enteral groups; ${ }^{\#} p<0.05$ and \& $p<0.001$ represent significance of the GLN intravenous + enteral group compared to GLN intravenous treatment. b Time course of GLN levels in portal circulation in GLN-treated control swine. Data are expressed as means \pm standard deviation. ${ }^{* *} p<0.01,{ }^{* * *} p<0.001$ represent significance of GLN intravenous, GLN intravenous + enteral and GLN enteral groups compared to the control portal group. ${ }^{\&} p<0.01$ and ${ }^{\#} p<0.001$ represent significance of the GLN intravenous + enteral group compared to the GLN enteral group; ${ }^{\S} p<0.001$ represents significance of the GLN enteral compared to the GLN intravenous group.

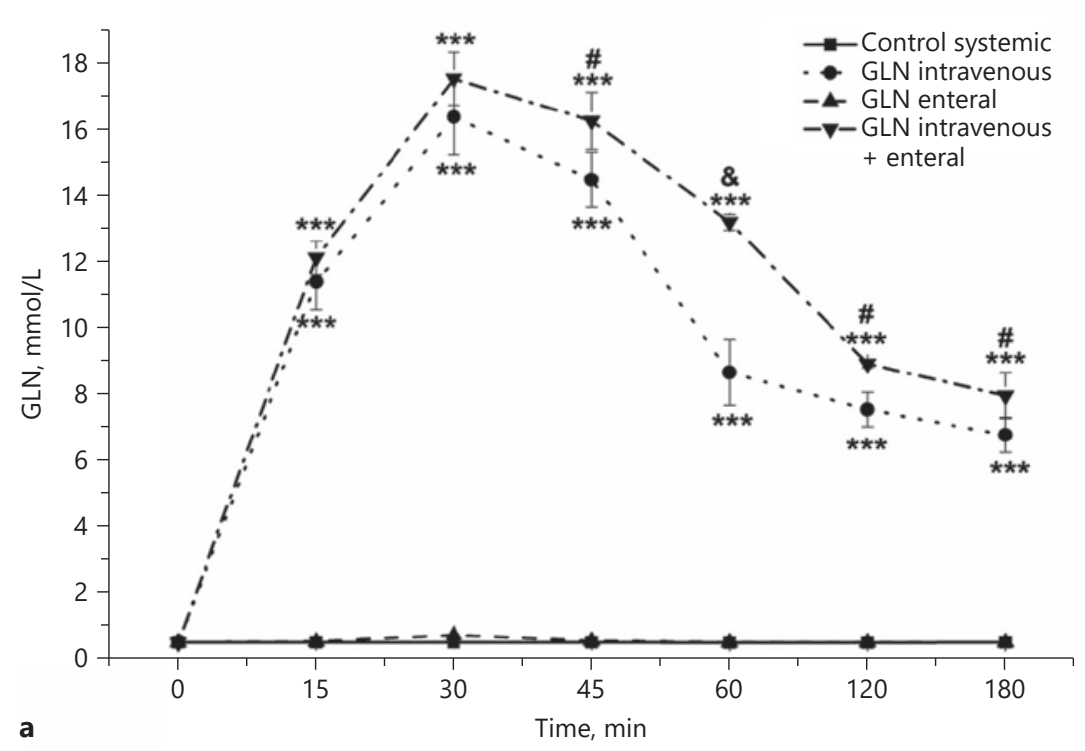

a

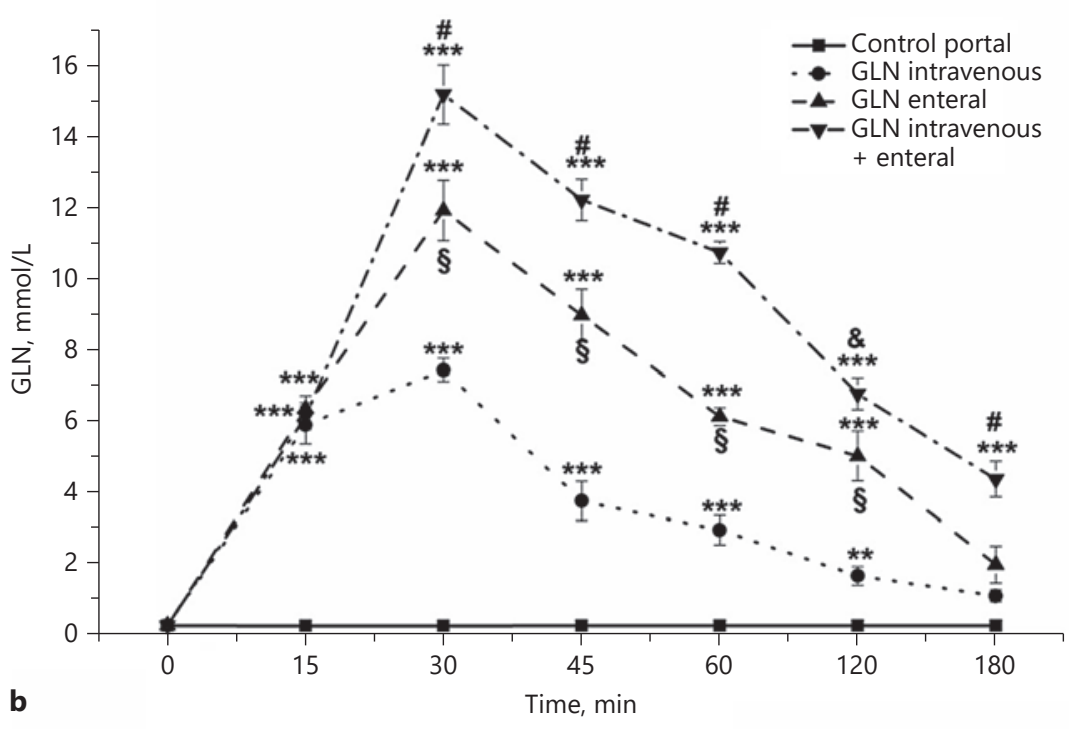

Table 2. Baseline and peak values ( $\mathrm{mmol} / \mathrm{L}$ ) of systemic and portal plasma GLN in healthy and endotoxemic swine

\begin{tabular}{|c|c|c|c|c|c|c|c|c|}
\hline & \multicolumn{4}{|c|}{ Healthy animals } & \multicolumn{4}{|c|}{ Endotoxemic animals } \\
\hline & \multicolumn{2}{|c|}{ systemic circulation } & \multicolumn{2}{|c|}{ portal circulation } & \multicolumn{2}{|c|}{ systemic circulation } & \multicolumn{2}{|c|}{ portal circulation } \\
\hline Intravenous & $16.3 \pm 1.13$ & $6.7 \pm 0.53$ & $7.5 \pm 0.33$ & $1.1 \pm 0.166$ & $4.2 \pm 0.19$ & $0.3 \pm 0.04$ & $2.5 \pm 0.11$ & $0.3 \pm 0.04$ \\
\hline Enteral & $0.7 \pm 0.03$ & $0.4 \pm 0.03$ & $12 \pm 0.85$ & $2 \pm 0.52$ & $0.4 \pm 0.02$ & $0.2 \pm 0.01$ & $0.4 \pm 0.006$ & $0.2 \pm 0.015$ \\
\hline
\end{tabular}


Fig. 3. a Time course of GLN levels in systemic circulation in GLN-treated endotoxemic (mentioned as "sepsis") swine. Data are expressed as means \pm standard deviation. ${ }^{* * *} p<0.001$ represents significance of sepsis GLN intravenous and sepsis GLN intravenous + enteral groups compared to all control systemic, sepsis systemic and sepsis GLN enteral groups; ${ }^{\#} p<0.01$ and $\& p<0.001$ represent significance of the sepsis GLN intravenous + enteral group compared to the sepsis GLN intravenous group. b Time course of GLN levels in portal circulation in GLN-treated endotoxemic (mentioned as "sepsis") swine. Data are expressed as means \pm standard deviation. ${ }^{* * *} p<0.001$ represents significance of sepsis GLN intravenous and sepsis GLN intravenous + enteral groups compared to all control portal, sepsis portal and sepsis GLN enteral groups; ${ }^{*} p<0.001$ represents significance of the sepsis GLN intravenous + enteral group compared to the sepsis GLN intravenous group; ${ }^{\&} p<0.05$ represents significance of the GLN enteral group compared to both control portal and sepsis portal groups.

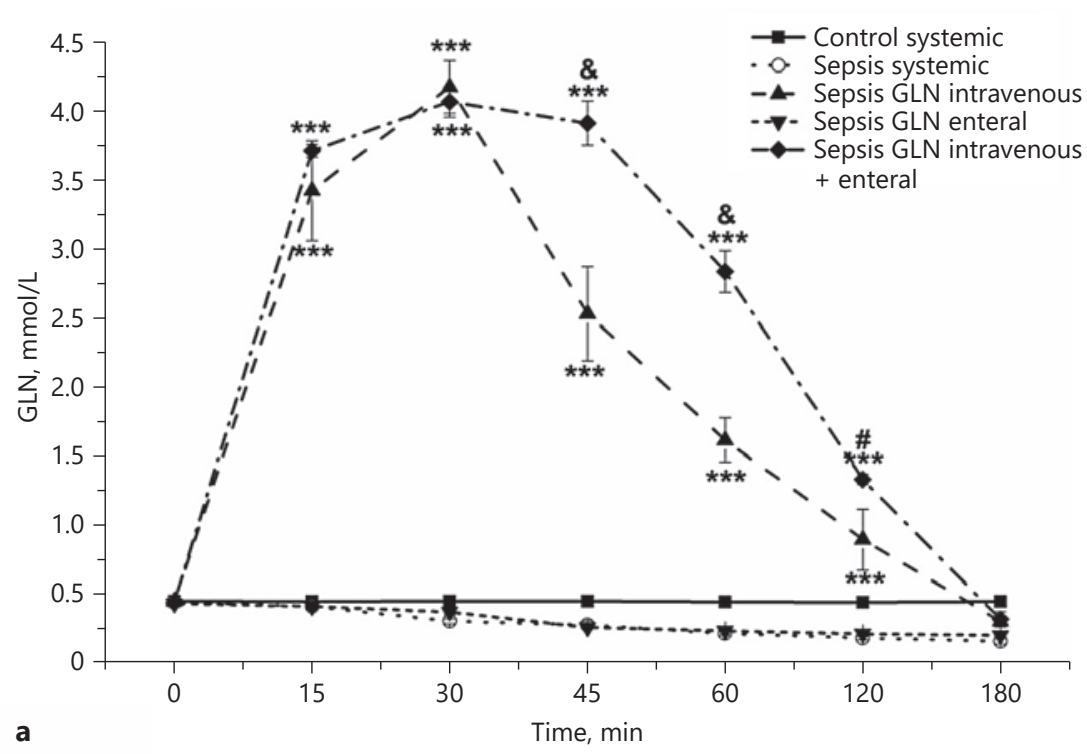

a

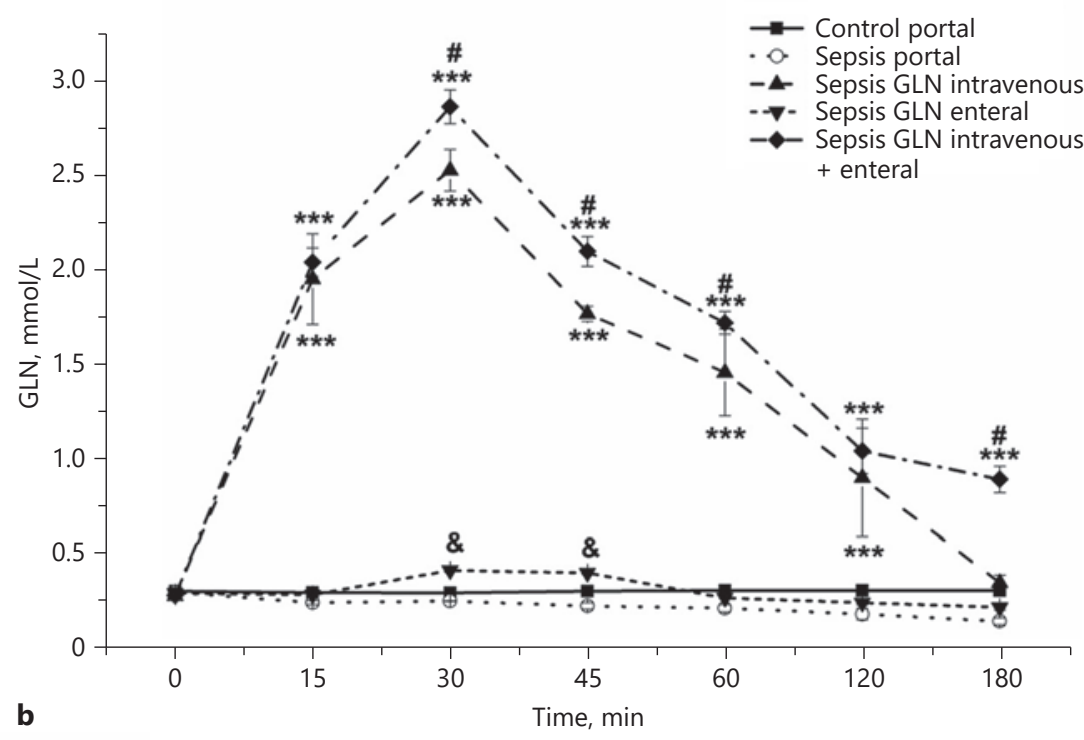

compared to baseline, $p<0.001)$. After enteral administration, only a slight $(1.5$-fold $)$ increase $(p<0.001)$ was found at $30 \mathrm{~min}$, followed by a progressive decline to baseline. After GLN had been given via both routes, a 40fold increase $(p<0.001)$ occurred at $30 \mathrm{~min}$, followed by a similar progressive elimination curve, reaching an 18fold increase at $180 \mathrm{~min}$. When intravenous-only and combined-infusion curves were analyzed, the latter approach achieved higher values $(p=0.006)$ from $30 \mathrm{~min}$ and thereafter (Table 2, Fig. 2a).
Similarly, intravenous GLN resulted in a 24.6-fold increase $(p<0.001)$ of portal levels at $30 \mathrm{~min}$, while 4 -fold greater values compared to baseline were found at 180 min $(p<0.001)$. Enteral infusion resulted in a 40 -fold increase $(p<0.001)$, but then declined progressively to 7 -fold greater levels $(p<0.001)$. After combined administration, a 52 -fold increase $(p<0.001)$ was found, keeping plasma values to a high (15-fold) level at $180 \mathrm{~min}$ $(p<0.001)$ (Table 2, Fig. 2b). As observed in systemic circulation, combined infusion resulted in the highest 
levels from 30 to $180 \mathrm{~min}$, compared to enteral administration alone, and to an even greater extent compared to intravenous infusion alone.

Based on the understanding that endogenous GLN exhibits a progressive decline during the 180 -min endotoxemia period, we correlated GLN levels in GLN-treated endotoxemic animals with both the baseline and the endogenous levels at each corresponding time point, in endotoxemic controls. After intravenous administration, a 9.5-fold increase $(p<0.001)$ was observed (30 $\mathrm{min})$; thereafter, a progressive decrease was prominent, in levels as low as the baseline $(180 \mathrm{~min})(p=0.024)$. When comparing these levels with the corresponding time point in endotoxemic controls, a 14-fold and a 2 -fold ( $p<0.001)$ increase was observed, at 30 and 180 min. Enteral GLN was found to maintain the declining GLN levels at slightly higher values than in endotoxemic controls, i.e., 1.2 -fold $(p=0.024)$ and 2.2 -fold $(p<0.001)$ decreases, at 30 and $180 \mathrm{~min}$, compared to baseline, and nonsignificant increases by 1.2 -fold and 1.3-fold at 30 and $180 \mathrm{~min}$, compared to the corresponding time point in endotoxemic controls. After combined administration a 9.3-fold increase $(p<0.001)$ was found at $30 \mathrm{~min}$; thereafter, a progressive decline resulted in 1.7-fold lower levels than baseline $(p<0.001)$. When values were compared with the corresponding time points in endotoxemic controls, levels at $30 \mathrm{~min}$ were increased by 14fold $(p=0.001)$ and those at $180 \mathrm{~min}$ by 2 -fold $(p=0.001)$. Finally, when intravenous-only and combined-infusion curves were analyzed, the latter approach achieved higher values $(p<0.001)$ at 45,60 and $120 \mathrm{~min}$ (Table 2, Fig. 3a).

With respect to portal circulation, endotoxemic animals treated intravenously exhibited a 9.4-fold increase at 30 min compared to baseline $(p<0.001)$, followed by a progressive decrease, leading to 1.3 -fold greater values at $180 \mathrm{~min}(p=0.045)$. When comparing these levels with the corresponding time point levels in endotoxemic controls, an 11-fold and a 2.5 -fold increase $(p<0.001)$ was evident at 30 and $180 \mathrm{~min}$. Enteral GLN caused only a slight increase (1.4-fold, $p<0.001$ ) during the 30 -min infusion compared to baseline, followed by a progressive decline to levels lower than the baseline at $180 \mathrm{~min}(p=$ $0.004)$. When comparing the corresponding values in endotoxemic controls, GLN levels were 1.7-fold $(p=0.036)$ and 1.6-fold higher at 30 and $180 \mathrm{~min}$ (Table 2, Fig. 3b).

We must emphasize that in healthy animals portal GLN levels after enteral administration were lower than those after combined use $(p<0.001$ at 30 and $180 \mathrm{~min})$ but higher than those after intravenous treatment $(p<$
0.001 at $30 \mathrm{~min}, p=0.026$ at $180 \mathrm{~min}$ ). In contrast, in endotoxemic animals, portal levels after enteral administration were at their lowest level, lower than after intravenous or combined GLN administration $(p<0.001$ at $30 \mathrm{~min}$ and $180 \mathrm{~min}$ ).

After combined treatment, an 11-fold increase $(p<$ 0.001 ) was found at $30 \mathrm{~min}$ compared to baseline, keeping plasma GLN values significantly higher at $180 \mathrm{~min}$ (3.3-fold, $p=0.003$ ). When compared to the corresponding levels in endotoxemic controls, values were 12 -fold and 6.6-fold higher $(p<0.001)$ at $30 \mathrm{~min}$ and $180 \mathrm{~min}$. As observed in systemic circulation, combined infusion resulted in the highest portal levels from 30 to $180 \mathrm{~min}$, compared to intravenous infusion alone $(p<0.001)$, and much more compared to enteral infusion alone $(p<$ $0.001)$. Finally, after combined administration, the 30 min levels peaked at $17.4 \pm 0.79$ and $15.2 \pm 0.84 \mathrm{mmol} / \mathrm{L}$ in healthy but only at $4.1 \pm 0.11$ and $2.9 \pm 0.09 \mathrm{mmol} / \mathrm{L}$ in endotoxemic animals, in systemic and portal circulation.

\section{Discussion}

The concept of combined parenteral and enteral GLN supplementation emerged as a potential way to achieve higher GLN levels in various tissues, in a short period of time. This concept takes into account the different exogenous GLN absorption pathways, according to the route of administration.

Accepting the assumption that following combined administration, the major proportion of the enteral GLN would be utilized by the enterocytes and immunocompetent cells, and the rest escaping into the portal vein by the liver, this would allow only a small fraction to appear in the periphery [7-9]. Consequently, the whole parenteral dose (plus that escaping from the gut/liver) would then be used to meet the needs of the rest of the body. Despite numerous publications, there is still limited evidence regarding systemic and portal circulation levels of GLN in sepsis and, in particular, in relation to the route of administration [7]. Thus, we decided to apply this experimental model to swine, since their gastrointestinal tract closely resembles that of humans [10]. We recorded the systemic and portal levels of GLN achieved after intravenous, enteral and combined supplementation compared to the baseline, as well as to placebo, at the same time points, throughout a 3-h study period. The corresponding levels in healthy and endotoxemic animals were compared. 
An important finding of the present experiment is related to endogenous GLN levels in healthy and endotoxemic swine, receiving no GLN treatment at all. GLN baseline levels were found to be similar to those in healthy humans (approx. $0.420 \mathrm{mmol} / \mathrm{L}$ ) [11], whereas after LPS administration a progressive decline was prominent, as supported by previous reports $[1,2,11]$. In our study all comparisons have been made in relation to both baseline and the time-point-matched measurement in endotoxemic animals.

With respect to healthy animals, this study clearly indicates that combined administration confers significant superiority over intravenous treatment alone, in terms of enhanced availability: a 30-min intravenous or combined infusion promoted a gradual increase in systemic circulation levels, which peaked at $30 \mathrm{~min}$ by as much as 36 -fold and 40-fold, respectively, followed by a gradual decrease, remaining 15-fold and 18-fold higher at $180 \mathrm{~min}$. This can be explained in two ways: either a small fraction escapes into the systemic circulation after enteral administration adding to that given intravenously, thus increasing plasma concentrations, or, when GLN is given intravenously, splanchnic consumption decreases, meaning the fraction, enterally given, escaping into the portal circulation increases, resulting in an increase in the total concentration measured in systemic circulation, compared to that obtained by the intravenous route alone, the latter being supported previously [12].

Likewise, in LPS-treated swine, the pattern of GLN changes in systemic circulation was similar in both intravenous and combined-infusion groups at $30 \mathrm{~min}$, possibly due to total utilization of enteral GLN by the splanchnic bed. At the end of the infusion, a progressive decline in GLN levels began, which was much more pronounced compared to healthy animals. GLN levels reached baseline at $180 \mathrm{~min}$, the value being 2 -fold higher compared to endogenous levels in endotoxemic controls, in the same period. Moreover, it should be noted that after the 30-min infusion, via either route, the elimination curve in systemic circulation remained at significantly higher levels after combined administration, compared to intravenous treatment alone. This occurred in both healthy and endotoxemic animals, but to different degrees.

Regarding portal GLN concentrations, arteriovenous measurements from an isolated intestine perfusion model showed that in each pass through the gut there was a net GLN extraction of about $170 \mu \mathrm{mol} / \mathrm{L}$ of plasma, or more than $20 \%$ of the total plasma content [13]. Simi- larly, portal-derived viscera as a whole utilize $167 \mu \mathrm{mol} /$ $\mathrm{kg} / \mathrm{h}$ [14], while adult rat enterocytes extract $25-33 \%$ of arterial GLN [15]. Furthermore, GLN is synthesized by the intestine, but at a lower rate than it is utilized, resulting in net uptake from the circulation in a dose-dependent manner; net uptake was equal to $60-80 \mu \mathrm{mol} / \mathrm{h}$ at concentrations above $600 \mu \mathrm{mol} / \mathrm{L}$ but decreased linearly to zero as the concentrations fell to $200 \mu \mathrm{mol} / \mathrm{L}$ [13]. This matches well the real scenario of GLN depletion during stressful conditions, when GLN stores have been exhausted. In such cases, the splanchnic bed is left with little, if any, GLN, leading to a nutritional gap in the maintenance of the gut mucosal barrier [16]. Our findings reveal that, when intravenous GLN was steadily infused for $30 \mathrm{~min}$, both the systemic and portal levels progressively increased from 0.5 to $16.3 \mathrm{mmol} / \mathrm{L}$ and from 0.3 to 7.5 $\mathrm{mmol} / \mathrm{L}$ in healthy animals, while in endotoxemia, this was from 0.4 to only $4.2 \mathrm{mmol} / \mathrm{L}$ and from 0.3 to 2.5 $\mathrm{mmol} / \mathrm{L}$, respectively. Thereafter, a linear reduction was observed, which in endotoxemia reached the baseline within the following $130 \mathrm{~min}$.

The gut mucosa exhibits high net utilization of GLN; after enteral administration, $50-75 \%$ of GLN is metabolized by the viscera [11, 17-20]. The "first-pass elimination" ranges from 40 to $90 \%$ [21], while enterocytes metabolize a minimum of $600 \mu \mathrm{mol} / \mathrm{kg} / \mathrm{h}$ of diet-derived (enteral) glutamate - and, at the same time, the portaldrained viscera utilize $167 \mu \mathrm{mol} / \mathrm{kg} / \mathrm{h}$ of arterial GLN [14]. Our data confirm such findings: when GLN was given enterally, portal levels progressively increased 40 fold, from 0.3 to $12 \mathrm{mmol} / \mathrm{L}$ in healthy, but only 1.4 -fold (from 0.3 to $0.4 \mathrm{mmol} / \mathrm{L}$ ) in endotoxemic swine. This suggests that the endotoxemic gut either faces disproportionally greater metabolic needs or better absorbs GLN, when it is given enterally rather than parenterally, or both.

Regarding enteral absorption, when a GLN tracer was given enterally in healthy volunteers, almost three quarters were retained by the viscera [12]. When a large GLN load was infused simultaneously with the tracer, the relative amount of GLN retained by the splanchnic bed was reduced to $53 \%$ [12]. Our findings in endotoxemia support this finding; when $92 \mathrm{mmol}$ (13.5 g) [7] GLN was given intravenously, the gut was perfused through the mesenteric artery with an unknown fraction of these 92 $\mathrm{mmol}$, the highest fraction remaining in the portal vein (after utilization by the gut) being $2.5 \mathrm{mmol}$. When 92 mmol of GLN was given enterally, the gut was perfused through the lumen with the known quantity of $92 \mathrm{mmol}$, the highest portal fraction being only $0.4 \mathrm{mmol}$, and fi- 
nally, when $92 \mathrm{mmol}$ was given intravenously and another $92 \mathrm{mmol}$ enterally simultaneously, the highest portal fraction was $2.9 \mathrm{mmol}$, i.e., 7 -fold higher than the 0.4 mmol.

All the above findings, both in healthy swine and in the early endotoxemia model, support our suggestion that combined administration is more efficient in restoring plasma levels in both systemic and portal circulations.

Our study has several limitations. First, the rather short-term infusion, which was dictated by special experimental needs; we used a swine model of $E$. coli endotoxemia, administering GLN at the beginning and not before, in order to simulate the clinical condition. Moreover, we took into account that in this model the earlyendotoxemia phase (hyperdynamic - no need for inotropes) lasts 3-4 h [6], and treating hemodynamically unstable patients with GLN is considered a contraindication. It was thus necessary to gain time by shortening the infusion period. Second, the supramaximal dose used has already been applied clinically; intensive care unit patients were randomized to receive $0-0.86 \mathrm{~g} / \mathrm{kg} \mathrm{L}-\mathrm{GLN}$ in order to investigate the relation of the dose to muscle concentration [22], while $0.65 \mathrm{~g} / \mathrm{kg}$ was considered the maximally tolerated oral dose in children [23]. Third, contrary to existing recommendations $[24,25]$, GLN was provided without any concomitant nutritional support. This could be justified by the short duration of the protocol and the good nutritional status of the animals. Fourth, we assessed only plasma levels of GLN, and plas$\mathrm{ma}$ is a poor indicator of the intracellular depletion occurring in critical illness [22].

\section{Conclusion}

The findings of this experiment indicate that combined administration of GLN confers significant superiority over intravenous treatment alone, in terms of enhanced availability in systemic and portal circulation. Enteral GLN alone has a negligible effect on the systemic circulation, while it seems sufficient for the increased intestinal metabolic needs. Intravenous supplementation results in a uniform provision across the viscera, similar to the endogenous GLN. Thus, combined administration, at the onset of endotoxemia, emerges as a beneficial practice, ensuring adequate GLN to compensate for the forthcoming intracellular shortage. Obviously, the possible beneficial transfer of this practice to critically ill patients should be re-evaluated in well-designed, large-scale clinical trials, under strict evaluation of pretreatment GLN levels and under careful selection of patients, since experimental endotoxemia is so close and yet so far from human sepsis and multiorgan failure.

\section{Statement of Ethics}

The experimental protocol was approved by the Department of Animal Care and Use Committee of the Greek Ministry of Agriculture, according to the European Community Guiding Principles for the Care and Use of Animals (EU Directive 2010/63/EU, Protocol No. 164909/11.05.2012).

\section{Disclosure Statement}

The authors declare that they have no conflict of interest.

\section{References}

1 Gamrin L, Essén P, Forsberg AM, Hultman E, Wernerman J. A descriptive study of skeletal muscle metabolism in critically ill patients: free amino acids, energy-rich phosphates, protein, nucleic acids, fat, water, and electrolytes. Crit Care Med. 1996 Apr;24(4):575-83.

2 Rodas PC, Rooyackers O, Hebert C, Norberg $\AA$, Wernerman J. Glutamine and glutathione at ICU admission in relation to outcome [Lond]. Clin Sci (Lond). 2012 Jun;122(12): 591-7.

3 Kao C, Hsu J, Bandi V, Jahoor F. Alterations in glutamine metabolism and its conversion to citrulline in sepsis. Am J Physiol Endocrinol Metab. 2013 Jun;304(12):E1359-64.

4 de Oliveira GP, Silva JD, de Araújo CC, Prota LF, Abreu SC, Madeira C, et al. Intravenous glutamine administration reduces lung and distal organ injury in malnourished rats with sepsis. Shock. 2014 Mar;41(3):222-32.

5 Carrer DP, Kotzampassi K, Fyntanidou B, Grosomanidis V, Papapetropoulos A, Lymperi $\mathrm{M}$, et al. Modulation of the release of Ang-2 in experimental endotoxic shock by a species-specific circulating factor. Injury. 2013 Jul;44(7):935-40.

6 Kazamias P, Kotzampassi K, Koufogiannis D, Eleftheriadis E. Influence of enteral nutritioninduced splanchnic hyperemia on the septic origin of splanchnic ischemia. World J Surg. 1998 Jan;22(1):6-11.

7 Melis GC, Boelens PG, van der Sijp JR, Popovici T, De Bandt JP, Cynober L, et al. The feeding route (enteral or parenteral) affects the plasma response of the dipetide Ala-Gln and the amino acids glutamine, citrulline and ar- ginine, with the administration of Ala-Gln in preoperative patients. Br J Nutr. 2005 Jul; 94(1):19-26.

8 Hall JC, Dobb G, Hall J, de Sousa R, Brennan L, McCauley R. A prospective randomized trial of enteral glutamine in critical illness. Intensive Care Med. 2003 Oct;29(10):1710-6.

9 Wernerman J. Clinical use of glutamine supplementation. J Nutr. 2008 Oct;138(10):2040S-4S.

10 Gonzalez LM, Moeser AJ, Blikslager AT. Porcine models of digestive disease: the future of large animal translational research. Transl Res. 2015 Jul;166(1):12-27.

11 Oudemans-van Straaten HM, Bosman RJ, Treskes M, van der Spoel HJ, Zandstra DF. Plasma glutamine depletion and patient outcome in acute ICU admissions. Intensive Care Med. 2001 Jan;27(1):84-90. 
12 Hankard RG, Darmaun D, Sager BK, D'Amore D, Parsons WR, Haymond M. Response of glutamine metabolism to exogenous glutamine in humans. Am J Physiol. 1995 Oct;269(4 Pt 1):E663-70.

13 Windmueller HG, Spaeth AE. Uptake and metabolism of plasma glutamine by the small intestine. J Biol Chem. 1974 Aug;249(16): 5070-9.

14 Reeds PJ, Burrin DG, Jahoor F, Wykes L, Henry J, Frazer EM. Enteral glutamate is almost completely metabolized in first pass by the gastrointestinal tract of infant pigs. Am J Physiol. 1996 Mar;270(3 Pt 1):E413-8.

$15 \mathrm{Wu}$ G. Intestinal mucosal amino acid catabolism. J Nutr. 1998 Aug;128(8):1249-52.

16 McCauley R, Kong SE, Hall J. Glutamine and nucleotide metabolism within enterocytes. JPEN J Parenter Enteral Nutr. 1998 Mar-Apr; 22(2):105-11.
17 Matthews DE, Marano MA, Campbell RG. Splanchnic bed utilization of glutamine and glutamic acid in humans. Am J Physiol. 1993 Jun;264(6 Pt 1):E848-54.

18 Boza JJ, Maire J, Bovetto L, Ballèvre O. Plasma glutamine response to enteral administration of glutamine in human volunteers (free glutamine versus protein-bound glutamine) [free glutamine versus protein-bound glutamine]. Nutrition. 2000 Nov-Dec;16(11-12):1037-42.

19 Haisch M, Fukagawa NK, Matthews DE. Oxidation of glutamine by the splanchnic bed in humans. Am J Physiol Endocrinol Metab. 2000 Apr;278(4):E593-602.

20 Boza JJ, Dangin M, Moënnoz D, Montigon F, Vuichoud J, Jarret A, et al. Free and proteinbound glutamine have identical splanchnic extraction in healthy human volunteers. Am J Physiol Gastrointest Liver Physiol. 2001 Jul; 281(1):G267-74.
21 Wernerman J. Glutamine supplementation. Ann Intensive Care. 2011 Jul;1(1):25.

22 Tjäder I, Rooyackers O, Forsberg AM, Vesali RF, Garlick PJ, Wernerman J. Effects on skeletal muscle of intravenous glutamine supplementation to ICU patients. Intensive Care Med. 2004 Feb;30(2):266-75.

23 Ward E, Picton S, Reid U, Thomas D, Gardener C, Smith M, et al. Oral glutamine in paediatric oncology patients: a dose finding study. Eur J Clin Nutr. 2003 Jan;57(1):31-6.

24 Chen QH, Yang Y, He HL, et al. The effect of glutamine therapy on outcomes in critically ill patients: A meta-analysis of randomized controlled trials. Crit Care. 2014;18(1):R8.

25 McRae MP: Therapeutic benefits of glutamine: An umbrella review of meta-analyses. Biomed Rep. 2017;6(5):576-84. 